\title{
Expression of growth-regulated oncogene-1, hepatocyte growth factor, platelet-derived growth factor-AA and soluble E-selectin and their association with high-risk human papillomavirus infection in squamous cell carcinoma of the uterine cervix
}

\author{
YE ZHANG ${ }^{1,2}$, JIAN-ZHONG WU ${ }^{2}$, YONG-QIN YANG ${ }^{3}$, RONG MA ${ }^{2}$, JUN-YING ZHANG ${ }^{4}$ and JI-FENG FENG ${ }^{1}$ \\ ${ }^{1}$ Department of Chemotherapy, ${ }^{2}$ Research Center of Clinical Oncology and ${ }^{3}$ Department of Radiotherapy, \\ The Affiliated Jiangsu Cancer Hospital, Nanjing Medical University, Nanjing, Jiangsu 210009; \\ ${ }^{4}$ Department of Oncology, Xuzhou Medical College, Xuzhou, Jiangsu 221000, P.R. China
}

Received November 18, 2013; Accepted May 15, 2014

DOI: $10.3892 / \mathrm{mmr} .2014 .2293$

\begin{abstract}
The aim of the present study was to evaluate the clinical significance and prognostic value of growth-regulated oncogene-1 (GRO-1), hepatocyte growth factor (HGF), platelet-derived growth factor-AA (PDGF-AA), soluble E-selectin (sE-selectin) and high-risk human papillomavirus (HPV; types 16, 18/45, 31 and 33/52/58/67) infection in cervical squamous cell carcinoma (CSCC). A total of 426 cases were enrolled in the present study, of which 292 cases were patients with CSCC, 43 were patients with cervical intraepithelial neoplasia (CIN) and 91 were healthy controls. Luminex xMAP technology was used to detect the serum levels of GRO-1, HGF, PDGF-AA and sE-selectin in all cases and two-channel fluorescence quantitative polymerase chain reaction was used to determine HPV DNA in cervical scrapings from CSCC and CIN patients. The results demonstrated that the serum levels of GRO-1, HGF and sE-selectin were significantly higher in patients with CSCC compared with patients with CIN and the healthy controls $(\mathrm{P}<0.0001)$. Compared with the CIN patients, the HPV positive rate in the CSCC patients significantly increased $(\mathrm{P}=0.013)$. The four factors were correlated with certain clinicopathological variables of CSCC patients to a certain degree $(\mathrm{P}<0.05)$ and the levels of HGF were closely associated with HPV infection $(\mathrm{P}=0.039)$. The receiver operating characteristic curves demonstrated that HGF obtained the highest diagnostic value compared with
\end{abstract}

Correspondence to: Dr Ji-Feng Feng, Department of Chemotherapy, The Affiliated Jiangsu Cancer Hospital, Nanjing Medical University, 42 Baizi Ting Road, Xuanwu, Nanjing, Jiangsu 210009, P.R. China

E-mail: zhangye881221@163.com

Key words: cervical squamous cell carcinoma, growth-regulated oncogene-1, hepatocyte growth factor, platelet-derived growth factor-AA, soluble E-selectin, Luminex the other three factors. Multivariate Cox regression analysis demonstrated that the serum levels of HGF $(\mathrm{P}<0.0001)$, FIGO stage $(\mathrm{P}<0.0001)$ and pelvic lymph node metastasis $(\mathrm{P}=0.001)$ were independent prognostic factors in patients with CSCC, while high-risk HPV infection did not show any significance in this analysis. These results demonstrated that HGF may be a useful prognostic biomarker rather than high-risk HPV types in patients with CSCC.

\section{Introduction}

In recent years, cervical cancer has become the third most common female cancer and in 2008 there were $\sim 529,000$ new cases worldwide (1). Cervical squamous cell carcinoma (CSCC) is the most common pathological type of cervical cancer followed by cervical adenocarcinoma and adenosquamous carcinoma (2). The association between human papillomavirus (HPV) infection and the occurrence of cervical cancer has been verified by numerous studies $(3,4)$. HPV types 16,18 , $58,33,52,45,31$ and 35 are the most common types in cervical cancer patients from Asia (5). However, the prognostic value of HPV in cervical cancer remains controversial. Certain studies have demonstrated an association between HPV 18-related cervical cancer and a poor prognosis $(6,7)$. However, in previous studies, HPV-positive cervical cancer patients demonstrated a more favorable prognosis than HPV-negative patients $(8,9)$. Whereas certain authors were unable to find any significance between HPV types and their prognosis (10). Thus, the aim of the present study was to identify potent molecular markers associated with the prognosis of CSCC.

Growth-regulated oncogene-1 (GRO-1) is a member of the CXC chemokine family which contains an ELR motif (Glu-Leu-Arg) at the NH2 terminal and is important in angiogenesis (11). Overexpression of the GRO gene was initially identified in transformed Chinese hamster cells (12). An elevated expression of GRO-1 was detected in numerous malignancies $(13,14)$. Hepatocyte growth factor (HGF) was first identified as a type of mitogen extracted from hepatectomized rats in 1984 (15). The HGF/c-Met signaling pathway 
composed of HGF and its functional receptor c-Met promotes tumorigenesis, progression and tumor cell invasion (16). Platelet-derived growth factor-AA (PDGF-AA) is a member of the PDGF family which can promote the production of angiogenic factors, resulting in tumor angiogenesis (17). E-selectin is a member of the selectin family which is a family of cellular adhesion molecules contributing to cell-cell adhesion and is associated with tumor progression and metastasis. Soluble E-selectin (sE-selectin) can be used as a biomarker in the serum to detect and monitor the occurrence and development of cancer (18).

The roles of serum levels of GRO-1, HGF, PDGF-AA and sE-selectin as biomarkers have not yet been elucidated compared with the important roles of high-risk HPV in CSCC. Studies on these four factors in cervical cancer are rare. The aim of the present study was to analyze the clinical significance and prognostic value of these four factors and high-risk HPV infection in CSCC.

\section{Materials and methods}

Patients. A total of 292 females with CSCC, 43 females with cervical intraepithelial neoplasia (CIN) and 91 healthy age-matched females were recruited for physical examination from the Jiangsu Cancer Hospital (Nanjing, China) between February 2010 and March 2013. In total, 292 cases with CSCC were histopathologically confirmed as CSCC for the first time and did not undergo any treatment prior to collecting the specimens. All the CSCC patients were staged on the basis of the latest International Federation of Gynecology and Obstetrics (FIGO) staging. The characteristics of all the cases are shown in Table I.

Collection of the specimens. Fasting blood ( $3 \mathrm{ml})$ specimens were obtained from 335 patients and 91 healthy females. The blood specimens were immediately transferred into the test tubes and then centrifuged at 1,000 x $\mathrm{g}$ for $10 \mathrm{~min}$ and the serum was extracted. The serum samples were stored at $-80^{\circ} \mathrm{C}$ until detection.

A routine gynecological examination was performed on 292 females with CSCC and 43 females with CIN. The cervical secretions were washed using sterile physiological cotton balls and then cervical scrapings were collected from the squamous cell junction of the cervical canal using cotton swabs. Subsequently, they were placed in sterile glass tubes and stored at $-20^{\circ} \mathrm{C}$ until use.

The present study was approved by the Ethics Committee of Jiangsu Cancer Hospital and written informed consent was obtained from all the participants in accordance with the ethical standards laid down in the 1964 Declaration of Helsinki and its later amendments.

Luminex xMAP technology. Serum levels of GRO-1, HGF, PDGF-AA and sE-selectin were quantified using Luminex xMAP technology. The Human Cytokine/Chemokine kit (cat no. MPXHCYTO60KPMX42) was used for the detection of GRO-1 and PDGF-AA, and Human Circulating Cancer Biomarker Magnetic Bead Panel 1 (cat no. HCCBP1MAG-58K) was used for the detection of HGF. Levels of sE-selectin were determined with Human Cardiovascular Disease (CVD)
Panel 1 (cat no. HCVD1-67AK). All the kits were supplied by Millipore (Billerica, MA, USA) while the FLEXMAP 3D ${ }^{\mathrm{TM}}$ system was purchased from Luminex Corporation (Austin, TX, USA).

The main experimental steps were as follows (in strict accordance with the manufacturer's instructions): Firstly, standard and quality controls were prepared in accordance with the manufacturer's instructions, then $25 \mu \mathrm{l}$ serum and $25 \mu \mathrm{l}$ beads (Millipore) were added to the plate, following being incubated for agitation overnight at $4^{\circ} \mathrm{C}$. Subsequently, $25 \mu \mathrm{l}$ of rabbit anti-rat monoclonal antibodies were added into each well followed by incubation for $1 \mathrm{~h}$ at $20-25^{\circ} \mathrm{C}$. Streptavidin-phycoerythrin $(25 \mu \mathrm{l})$ was added to the well and the plate followed by incubation for $30 \mathrm{~min}$ at $20-25^{\circ} \mathrm{C}$. Following being washed twice, $100 \mu 1$ of sheath fluid (Millipore) was added to every well. Finally, the median fluorescence intensity data was analyzed on the Luminex analyzer (FLEXMAP 3D ${ }^{\mathrm{TM}}$ system; Luminex Corporation) and the final concentrations of the four factors were calculated using the weighted 5-parameter logistic method.

DNA extraction and quantitative polymerase chain reaction ( $q P C R)$. The steps for DNA extraction of cervical scrapings were as follows: Firstly, the cervical scrapings were mixed with $1.5 \mathrm{ml}$ sterile saline in a glass tube, then all the liquid was transferred into a $1.5 \mathrm{ml}$ microcentrifuge tube to be centrifuged at $12,000 \mathrm{x} \mathrm{g}$ for $10 \mathrm{~min}$. The supernatant was removed and the precipitate was digested using proteinase $\mathrm{K}$ (Omega Bio-Tek Inc., Norcross, GA, USA). Finally, DNA was extracted using chloroform. A Real quality RQ-HPV HR kit (AB ANALITICA, Padova, Italy) was used to identify and quantify the HPV DNA type (16, 18/45, 31 and 33/52/58/67) on the ABI 7300 Real-Time PCR system (Applied Biosystems, Foster City, CA, USA) and $\beta$-globin served as the internal control. qPCR was conducted in a $20 \mu \mathrm{l}$ volume containing $10 \mu \mathrm{l}$ AB Real Time mix (AB ANALITICA), $0.4 \mu$ l primer mix, $0.8 \mu 1$ probe mix, $6.8 \mu 1 \mathrm{H}_{2} \mathrm{O}$ and $2 \mu \mathrm{l}$ DNA. The PCR program comprised 40 cycles of $2 \mathrm{~min}$ at $50^{\circ} \mathrm{C}, 3 \mathrm{~min}$ at $95^{\circ} \mathrm{C}$, $15 \mathrm{sec}$ at $94^{\circ} \mathrm{C}$ and $40 \mathrm{sec}$ at $57^{\circ} \mathrm{C}$. When the quantitative value of HPV was $\geq 100$ gene copies, the result was positive for this type of HPV, otherwise the result was negative. All the experiments were repeated three times.

Statistical analysis. The concentrations of GRO-1, HGF, PDGF-AA and sE-selectin were not normally distributed, thus the Mann-Whitney U test and Kruskal-Wallis test were used in the present study, while the $\chi^{2}$ test was used to compare the positive rate of HPV between the groups. The correlations between the biomarkers were analyzed using Spearman's rank correlation coefficient. The areas under the receiver operating characteristic curves (AUROC) were used to evaluate the diagnostic accuracy. Youden's index [Youden's index $=$ sensitivity + specificity) -1$]$ is an indicator of authenticity of diagnostic tests and the optimal cut-off values were calculated when the Youden index was maximum. Overall survival time (OS) was calculated from the day of diagnosis to mortality due to cervical cancer or the latest follow-up, while progression-free survival (PFS) was calculated from the day of diagnosis to the initial disease progression or mortality from cervical cancer or the latest follow-up. The 
Table I. Characteristics of all the cases.

\begin{tabular}{|c|c|c|c|}
\hline \multirow[b]{2}{*}{ Characteristic } & \multicolumn{2}{|c|}{ Patients with } & \multirow[b]{2}{*}{ Healthy controls $n(\%)$} \\
\hline & $\operatorname{CSCC} \mathrm{n}(\%)$ & CIN n $(\%)$ & \\
\hline Age, median (range) & $48(27-84)$ & $45(29-61)$ & $43(33-72)$ \\
\hline \multicolumn{4}{|c|}{ FIGO Stage or CIN classification } \\
\hline I & $40(13.70)$ & $12(29.91)$ & \\
\hline II & $108(36.99)$ & $18(41.86)$ & \\
\hline III & $115(39.38)$ & $13(30.23)$ & \\
\hline IV & $29(9.93)$ & & \\
\hline \multicolumn{4}{|l|}{ Metastasis } \\
\hline Positive & $29(9.93)$ & & \\
\hline Negative & $263(90.07)$ & & \\
\hline \multicolumn{4}{|l|}{ Grading } \\
\hline 1 & $43(14.73)$ & & \\
\hline 2 & $147(50.34)$ & & \\
\hline 3 & $102(34.93)$ & & \\
\hline \multicolumn{4}{|l|}{ Pelvic nodal status } \\
\hline Positive & $150(51.37)$ & & \\
\hline Negative & $142(48.63)$ & & \\
\hline \multicolumn{4}{|l|}{ Tumor size } \\
\hline$>4 \mathrm{~cm}$ & $144(49.32)$ & & \\
\hline$\leq 4 \mathrm{~cm}$ & $148(50.68)$ & & \\
\hline Total number & $292(100)$ & $43(100)$ & $91(100)$ \\
\hline
\end{tabular}

CSCC, cervical squamous cell carcinoma; CIN, cervical intraepithelial neoplasia; FIGO, International Federation of Gynecology and Obstetrics.

Kaplan-Meier survival curves and the log-rank test were used to compare the differences between groups. Multivariate Cox regression analysis was used to screen out the independent prognostic variables. The median (25-75 percentiles) was used to describe the measurement data and $\mathrm{P}<0.05$ was considered to indicate a statistically significant difference. All the above statistical analyses was performed using SPSS version 21.0 (IBM, Armonk, NY, USA).

\section{Results}

Concentrations of GRO-1, HGF, PDGF-AA and sE-selectin in CSCC patients, CIN patients and the healthy controls. The serum levels of GRO-1, HGF and sE-selectin in patients with CSCC significantly increased compared with the CIN patients and the healthy controls [GRO-1: $748.43(501.49-1088.23$ pg/ml) vs. 620.07 $(386.60-846.58 \mathrm{pg} / \mathrm{ml})$ and $564.91(376.34-899.03 \mathrm{pg} / \mathrm{ml})$, $\mathrm{P}<0.0001$; HGF: 724.35 (501.68-1049.35 pg/ml) vs. 555.68 (409.21-711.13 pg/ml) and 476.27 (356.36-834.40 pg/ml), $\mathrm{P}<0.0001$; sE-selectin: 22176.68 (13767.43-34829.97 pg/ml) vs. $17214.00(10854.60-25306.98 \mathrm{pg} / \mathrm{ml})$ and 18276.34 (9028.38-26154.20 pg/ml), $\mathrm{P}<0.0001]$ and no significant difference was identified between the CIN patients and the healthy controls $(\mathrm{P}>0.05)$. No statistical difference was identified in the serum levels of PDGF-AA among the three groups [13311.48 (8952.06-19040.87 pg/ml) vs. 12220.30 (7623.88-18438.83pg/ml)and11224.32(8756.27-17643.22pg/ml), $\mathrm{P}=0.21]$ (Fig. 1).

Positive rate of HPV types between the CSCC patients and the CIN patients. HPV infection was identified in 205 out of 292 (70.21\%) of the CSCC patients as well as 22 out of $43(51.16 \%)$ of the CIN patients. A significant difference was found between the two groups $\left(\chi^{2}=6.22, \mathrm{P}=0.013\right)$. Table II shows the distribution of the HPV types in the HPV-positive patients. It was revealed that HPV 16 was the most common genotype in the CSCC patients (50.24\%) and the CIN patients $(45.45 \%)$ infected with HPV 16 only. In addition, HPV 16 was the most common genotype in the CSCC patients $(66.34 \%)$ and the CIN patients $(59.09 \%)$ in the overall prevalence (Table II).

Association between HPV infection and clinicopathological characteristics in CSCC patients. As demonstrated in Table III, HPV infection was not associated with age, FIGO stage, metastasis, grading, pelvic lymph node metastasis or tumor size of the CSCC patients $(\mathrm{P}>0.05)$.

Association between serum levels of GRO-1, HGF, PDGF-AA and $s E$-selectin with clinicopathological variables and $H P V$ infection in CSCC patients. The serum levels of GRO-1 were closely associated with FIGO stage $(\mathrm{P}=0.001)$, metastasis $(\mathrm{P}<0.0001)$, pathological grading $(\mathrm{P}<0.0001)$ and pelvic lymph node metastasis $(\mathrm{P}=0.003)$. The serum levels of HGF were 
A

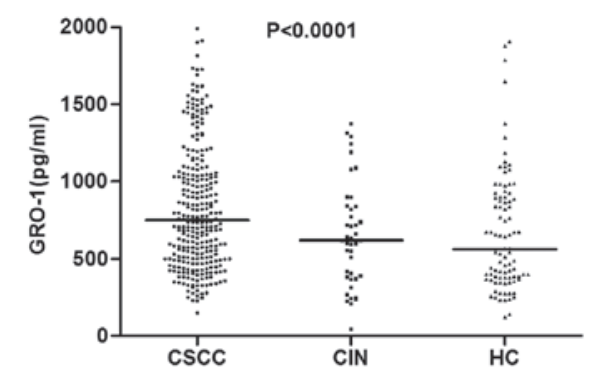

C

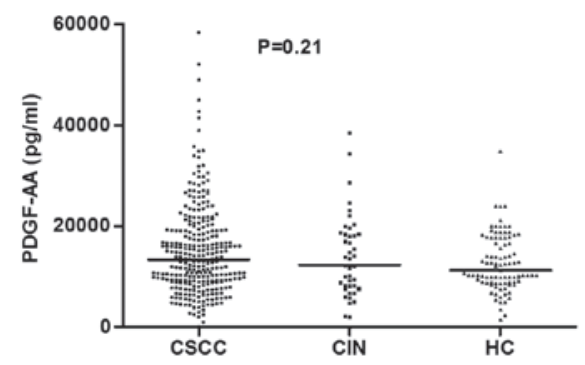

B

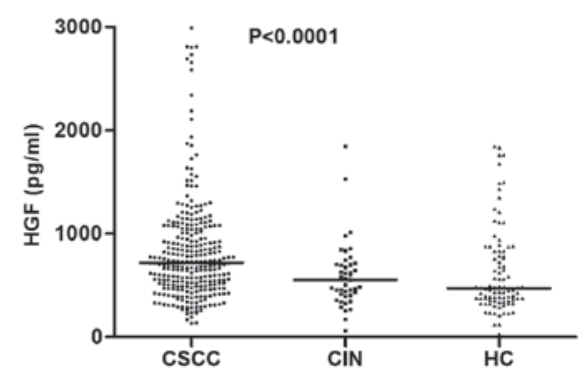

D

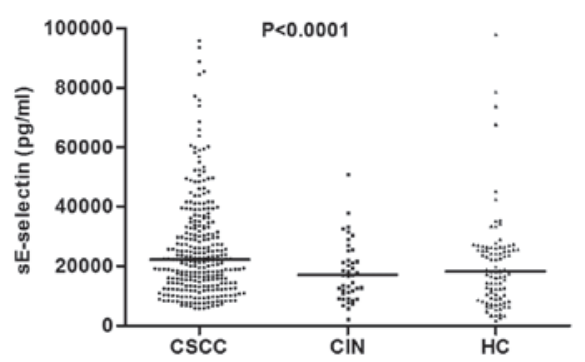

Figure 1. Concentrations of GRO-1, HGF, PDGF-AA and sE-selectin in CSCC patients, CIN patients and HCs. Each point represents an individual and the horizontal lines show the median values. (A) Concentration of GRO-1 in the three groups. (B) Concentration of HGF in the three groups. (C) Concentration of PDGF-AA in the three groups. (D) Concentration of sE-selectin in the three groups. CSCC, cervical squamous cell carcinoma; CIN, cervical intraepithelial neoplasia; HC, healthy controls; GRO-1, growth-regulated oncogene-1; HGF, hepatocyte growth factor; PDGF-AA, platelet-derived growth factor-AA; sE-selectin, soluble E-selectin.

Table II. Prevalence of HPV types in the HPV-positive patients.

\begin{tabular}{lrr}
\hline HPV type & $\begin{array}{c}\text { CSCC patients } \\
\mathrm{n}(\%)\end{array}$ & $\begin{array}{c}\text { CIN patients } \\
\mathrm{n}(\%)\end{array}$ \\
\hline 16 & $103(50.24)$ & $10(45.45)$ \\
$18 / 45$ & $17(8.29)$ & $5(22.73)$ \\
31 & $11(5.37)$ & $0(0.00)$ \\
$33 / 52 / 58 / 67$ & $35(17.07)$ & $4(18.18)$ \\
$16,18 / 45$ & $4(1.95)$ & $0(0.00)$ \\
16,31 & $4(1.95)$ & $0(0.00)$ \\
$16,33 / 52 / 58 / 67$ & $25(12.20)$ & $3(13.64)$ \\
$18 / 45,33 / 52 / 58 / 67$ & $4(1.95)$ & $0(0.00)$ \\
$31,33 / 52 / 58 / 67$ & $2(0.98)$ & $0(0.00)$ \\
Total infection number & $205(100)$ & $22(100)$ \\
\hline
\end{tabular}

CSCC, cervical squamous cell carcinoma; CIN, cervical intraepithelial neoplasia; HPV, human papillomavirus.

significantly correlated with FIGO stage $(\mathrm{P}=0.02)$, pathological grading $(\mathrm{P}<0.0001)$, pelvic lymph node metastasis $(\mathrm{P}<0.0001)$, tumor size $(\mathrm{P}<0.0001)$ and HPV infection $(\mathrm{P}=0.039)$. The concentrations of PDGF-AA in the serum were associated with FIGO stage $(\mathrm{P}=0.004)$, pathological grading $(\mathrm{P}=0.021)$ and pelvic lymph node metastasis $(\mathrm{P}<0.0001)$, and the concentrations of sE-selectin were correlated with age $(\mathrm{P}<0.0001)$, FIGO stage $(\mathrm{P}<0.0001)$ and tumor size $(\mathrm{P}=0.001)$ (Table IV).

Correlations between GRO-1, HGF, PDGF-AA and $s E$-selectin in patients with CSCC. Fig. 2 shows that there were several positive correlations of the serum levels of HGF with the other three factors: Serum levels of HGF correlated with GRO-1 ( $\mathrm{R}=0.194, \mathrm{P}=0.001)$, PDGF-AA $(\mathrm{R}=0.202, \mathrm{P}=0.0005)$ and sE-selectin $(\mathrm{R}=0.214, \mathrm{P}=0.0002)$. The concentration of PDGF-AA was correlated with GRO-1 $(\mathrm{R}=0.361, \mathrm{P}<0.0001)$, while no association of sE-selectin with GRO-1 $(\mathrm{R}=0.001$, $\mathrm{P}=0.991)$ and PDGF-AA ( $\mathrm{R}=-0.002, \mathrm{P}=0.971)$ was identified.

Diagnostic value of serum levels of GRO-1, HGF, PDGF-AA and sE-selectin in the CSCC patients. AUROC of GRO-1, HGF, PDGF-AA and sE-selectin for discriminating the CSCC patients from the CIN patients were 0.615 (95\% CI: $0.525-0.705$ ), 0.662 (95\% CI: $0.583-0.741), 0.537$ (95\% CI: $0.442-0.631$ ) and 0.634 (95\% CI: 0.551-0.718), respectively, while the AUROC of HGF for discriminating the CSCC patients from the healthy controls was 0.655 (95\% CI: 0.586-0.723) compared with 0.633 (95\% CI: 0.566-0.700) for GRO-1, 0.559 (95\% CI: 0.497-0.622) for PDGF-AA and 0.618 (95\% CI: 0.552-0.684) for sE-selectin. In addition, the AUROC of GRO-1, HGF, PDGF-AA and sE-selectin for discriminating the CSCC patients from the CIN patients and the healthy controls were 0.627 (95\% CI: 0.570-0.684), 0.657 (95\% CI: $0.600-0.714), 0.552$ (95\% CI: 0.495-0.609) and 0.624 (95\% CI: 0.567-0.680), respectively (Fig. 3). The results demonstrated that serum levels of HGF obtained the highest diagnostic value compared with the other three factors. Table V shows the sensitivity and specificity of the optimal cut-off values.

Prognostic value of GRO-1, HGF, PDGF-AA, sE-selectin and $H P V$ in CSCC patients. The median values of GRO-1, HGF, PDGF-AA and sE-selectin were used as the cut-off points. The log-rank analysis demonstrated that patients with a low expression of GRO-1 $(<748.43 \mathrm{pg} / \mathrm{ml})$ had significantly longer OS and PFS than those with a high expression of GRO-1 
Table III. Association between HPV and clinicopathological characteristics in 292 cervical squamous cell carcinoma patients.

\begin{tabular}{|c|c|c|c|c|c|}
\hline Characteristic & Patients (n) & HPV positive $\mathrm{n}(\%)$ & HPV negative n (\%) & $\chi^{2}$ & P-value \\
\hline \multicolumn{6}{|l|}{ Age (years) } \\
\hline$\leq 45$ & 175 & $121(69.14)$ & $54(30.86)$ & 0.236 & 0.627 \\
\hline$>45$ & 117 & $84(71.79)$ & $33(28.21)$ & & \\
\hline \multicolumn{6}{|l|}{ FIGO stage } \\
\hline $\mathrm{I}+\mathrm{II}$ & 148 & 99 (66.89) & $49(33.11)$ & 1.575 & 0.209 \\
\hline III+IV & 144 & $106(73.61)$ & $38(26.39)$ & & \\
\hline \multicolumn{6}{|l|}{ Metastasis } \\
\hline Positive & 29 & $20(68.97)$ & $9(31.03)$ & 0.024 & 0.878 \\
\hline Negative & 263 & $185(70.34)$ & 78 (29.66) & & \\
\hline \multicolumn{6}{|l|}{ Grading } \\
\hline 1 & 43 & $29(67.44)$ & $14(32.56)$ & 0.249 & 0.883 \\
\hline 2 & 147 & $103(70.07)$ & $44(29.93)$ & & \\
\hline 3 & 102 & 73 (71.57) & $29(28.43)$ & & \\
\hline \multicolumn{6}{|c|}{ Pelvic lymph node } \\
\hline Positive & 150 & $105(70.00)$ & $45(30.00)$ & 0.006 & 0.937 \\
\hline Negative & 142 & $100(70.42)$ & $42(29.58)$ & & \\
\hline \multicolumn{6}{|l|}{ Tumor size } \\
\hline$>4 \mathrm{~cm}$ & 144 & $99(68.75)$ & $45(31.25)$ & 0.288 & 0.592 \\
\hline$\leq 4 \mathrm{~cm}$ & 148 & $106(71.62)$ & $42(28.38)$ & & \\
\hline
\end{tabular}

HPV, human papillomavirus.

( $>748.43 \mathrm{pg} / \mathrm{ml}$; OS, $\mathrm{P}=0.049$; PFS, $\mathrm{P}=0.048)$, and that patients with a high expression of HGF ( $>724.35 \mathrm{pg} / \mathrm{ml})$ had significantly shorter OS and PFS than those with a low expression of HGF ( $<724.35 \mathrm{pg} / \mathrm{ml}$; OS, $\mathrm{P}<0.0001$; PFS, $\mathrm{P}<0.0001)$. The analysis also indicated significant differences in OS and PFS between patients with a high $(>22176.68 \mathrm{pg} / \mathrm{ml})$ and low expression $(<22176.68 \mathrm{pg} / \mathrm{ml})$ of sE-selectin (OS, $\mathrm{P}=0.048$; PFS, $\mathrm{P}=0.028)$. However, no differences in OS and PFS between patients with a high $(>13311.48 \mathrm{pg} / \mathrm{ml})$ and low expression $(<13311.48 \mathrm{pg} / \mathrm{ml})$ of PDGF-AA (OS, P=0.209; PFS, P=0.207) were identified (Fig. 4). Furthermore, HPV-positive patients had shorter OS and PFS compared with the HPV-negative patients (OS, $\mathrm{P}=0.027$; PFS, $\mathrm{P}=0.023$ ) (Fig. 5). Table VI indicated that only the expression of HGF in the serum $(\mathrm{P}<0.0001)$, FIGO stage $(\mathrm{P}<0.0001)$ and pelvic lymph node metastasis $(\mathrm{P}=0.001)$ were independent prognostic factors in CSCC patients, while high-risk HPV infection did not show any statistical significance in the multivariate Cox analysis.

\section{Discussion}

GRO-1, also termed CXCL1, has been extensively investigated in cancer in recent years (11-14). It is highly expressed in numerous types of carcinomas, including oropharyngeal cancer (19), colorectal cancer (13) and gastric cancer (14). Ogata et al (13) demonstrated that the expression of GRO-1 is associated with tumor size, staging, lymph node metastasis and invasion depth in colorectal cancer using immunohistochemistry. Jung et al (14) detected the serum levels of GRO-1 in gastric cancer and found that its high expression was corre- lated with tumor stage and lymph node metastasis. However, studies investigating this factor in CSCC are extremely rare. In the present study, the serum levels of GRO-1 in CSCC patients were significantly higher than patients with $\mathrm{CIN}$ and the healthy controls. In addition, the serum levels were associated with FIGO stage, metastasis, pathological grading and pelvic lymph node metastasis. In addition, the concentration of GRO-1 in patients whose tumor size was $>4 \mathrm{~cm}$ was higher than those in patients whose tumor size was $\leq 4 \mathrm{~cm}$, however, no difference was observed between them, and this may be due to the small sample size. The present study also could not verify the association between age and GRO-1. This result is consistent with several previous studies $(13,14)$. Overall, detecting the serum levels of GRO-1 in CSCC patients may be useful for predicting the state of tumor load and the progression of the disease.

Several studies have indicated that the expression of HGF mainly secreted by stromal cells is elevated in various types of carcinoma $(20,21)$, including cervical cancer (22). Certain studies have demonstrated that overexpression of HGF is associated with the progression and metastasis of hepatocellular carcinoma (20). Another study emphasized the important role of HGF and c-MET in the progression and invasion of esophageal squamous cell carcinoma (23). Nakamura et al (24) reported that HGF antagonists or inhibitors of c-MET were able to inhibit tumor growth and metastasis. The results from the present study demonstrated that serum levels of HGF in CSCC patients were significantly increased compared with the CIN patients and healthy controls, and that the serum levels were correlated with FIGO stage, pathological grade, pelvic 


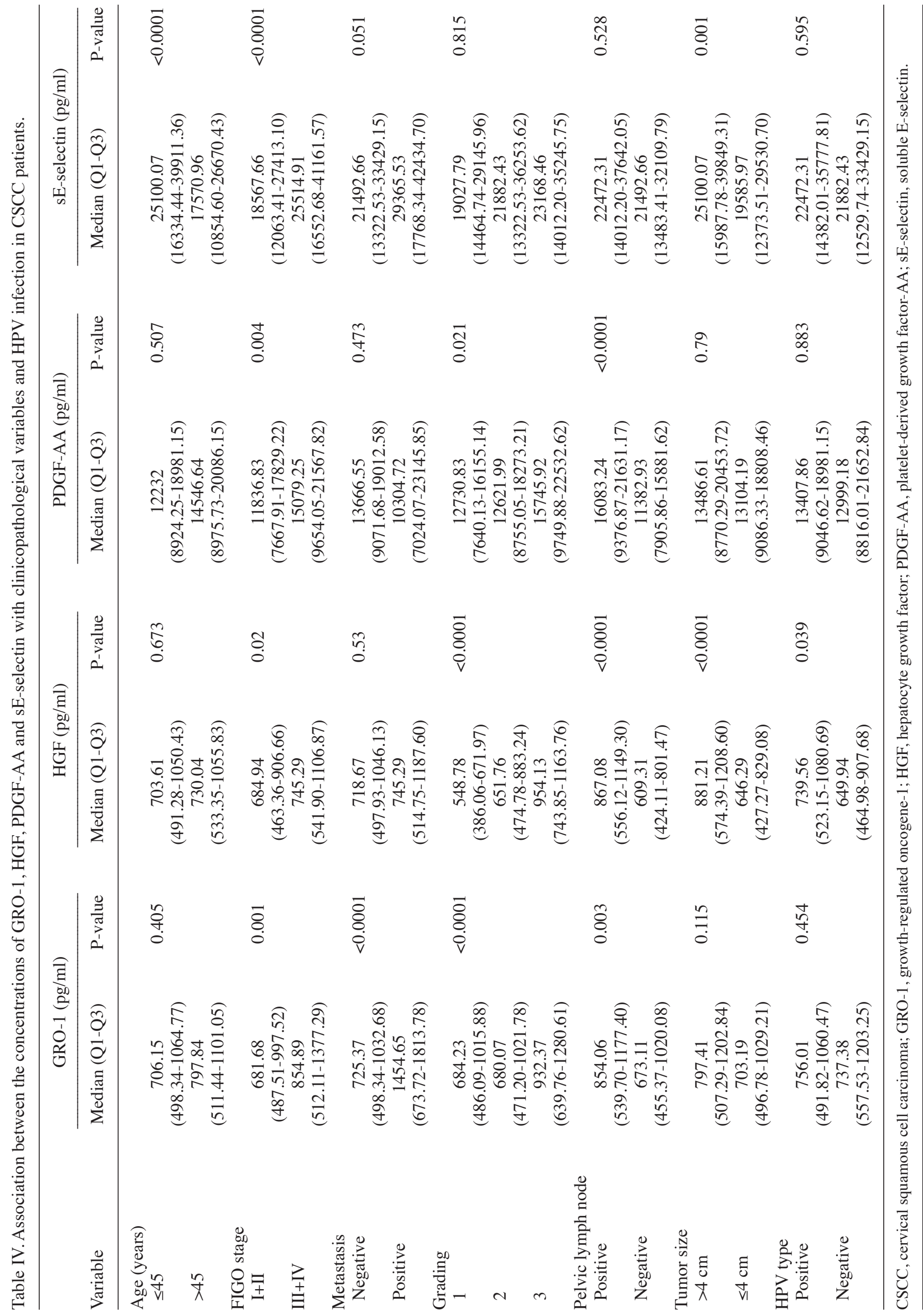


A

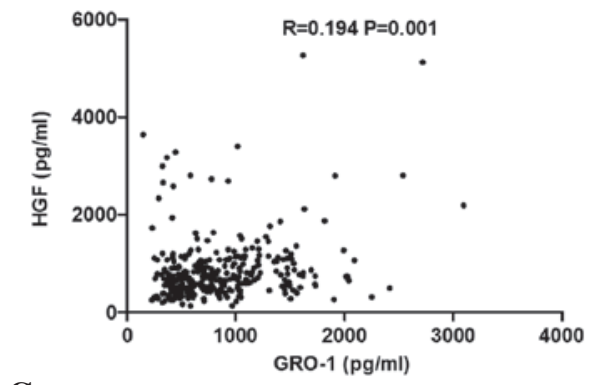

C

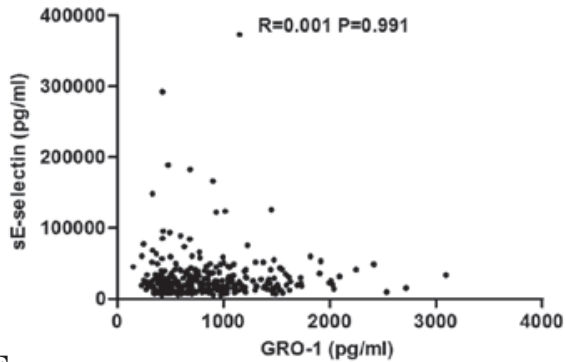

E

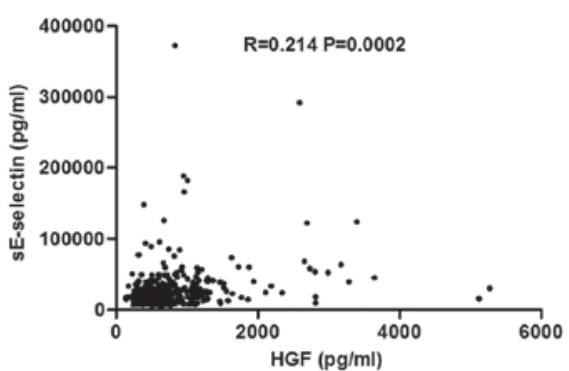

B

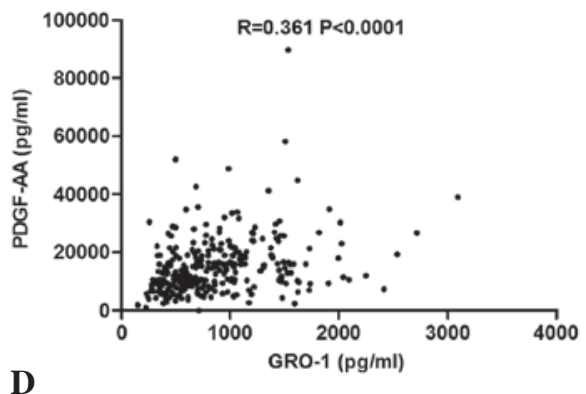

D

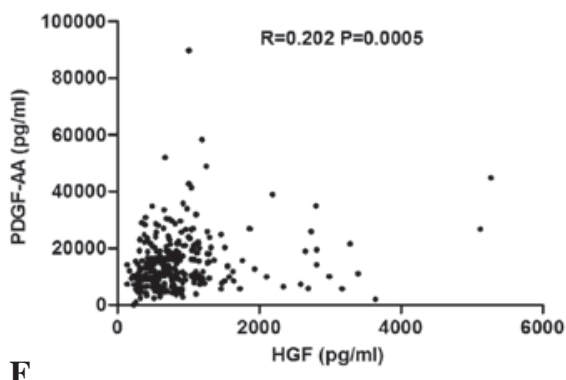

$\mathbf{F}$

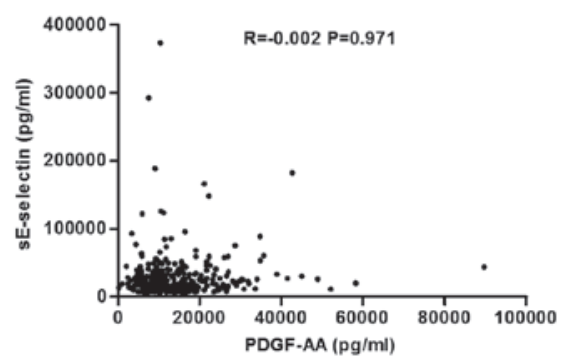

Figure 2. Scatter diagrams of the correlations between GRO-1, HGF, PDGF-AA and sE-selectin in CSCC patients. (A) Scatter diagram of the correlation between HGF and GRO-1 in CSCC patients. (B) Scatter diagram of the correlation between GRO-1 and PDGF-AA in CSCC patients. (C) Scatter diagram of the correlation between GRO-1 and sE-selectin in CSCC patients. (D) Scatter diagram of the correlation between HGF and PDGF-AA in CSCC patients. (E) Scatter diagram of the correlation between HGF and sE-selectin in CSCC patients. (F) Scatter diagram of the correlation between PDGF-AA and sE-selectin in CSCC patients. CSCC, cervical squamous cell carcinoma; GRO-1, growth-regulated oncogene-1; HGF, hepatocyte growth factor; PDGF-AA, platelet-derived growth factor-AA; sE-selectin, soluble E-selectin.

lymph node metastasis and tumor size. These findings suggest that the expression of HGF is associated with infiltration and tumor metastasis. Therefore, inhibiting the HGF/c-MET signaling pathway may be a potential therapeutic treatment for CSCC. In the present study, it was also observed that the serum levels of HGF in patients with metastasis were higher than those without metastasis, however, no statistical significance was observed between them. In addition, no correlation between HGF and age was found. Aune et al (25) demonstrated that preoperative serum HGF level is not associated with age, which is consistent with the results from the present study.

Several studies have demonstrated that the upregulation of PDGF in numerous carcinomas is able to promote lymph node metastasis and tumor cell proliferation $(26,27)$. Importantly, it can promote the formation of tumor blood vessels and lymph vessels $(28,29)$. Therefore, PDGF may stimulate the growth and metastasis of tumors by angiogenesis and lymphangiogenesis. It is well established that vascular endothelial growth factor (VEGF) is important in neoplasm angiogenesis. As a member of the PDGF family, PDGF-AA is an important autocrine regulator, which can regulate the expression of VEGF, thus PDGF-AA may be a more potent therapeutic target for inhibiting angiogenesis in tumors than VEGF (30). In the present study, it was observed that the concentrations of PDGF-AA in the serum of CSCC patients were correlated with FIGO stage, pathological grading and lymph node metastasis, and these results were in accordance with the above-mentioned studies. However, the results from the present study demonstrated that the serum levels of PDGF-AA were not associated with metastasis or tumor size and no association of PDGF-AA between the CSCC patients and the other two groups was found. The reasons for these results are diverse. Although PDGF-AA is a potent angiogenic factor, tumor angiogenesis is a multistep process including numerous growth factors and cytokines and every step may lead to different outcomes. The present study also verified that serum levels of PDGF-AA were not associated with age, which is consistent with a former study (26).

Previous studies have demonstrated that sE-selectin is highly expressed in colorectal cancer and is closely associated with the progression, recurrence and metastasis of cancer $(31,32)$. It was observed that the serum levels of sE-selectin were significantly increased in CSCC patients and were correlated with FIGO stage and tumor size. These findings suggested that the levels of sE-selectin can predict tumor invasion and progression. No association was found between sE-selectin and metastasis and certain studies also failed to 
A

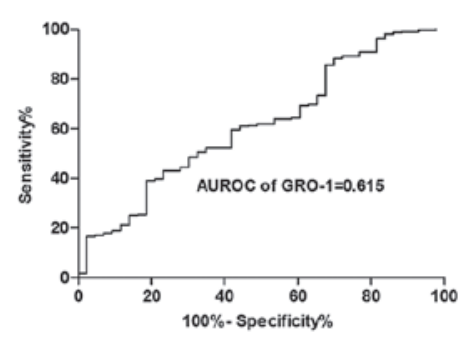

C

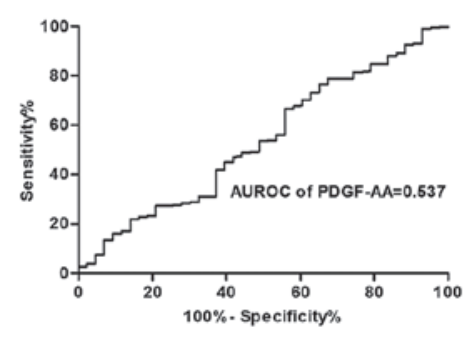

$\mathbf{E}$

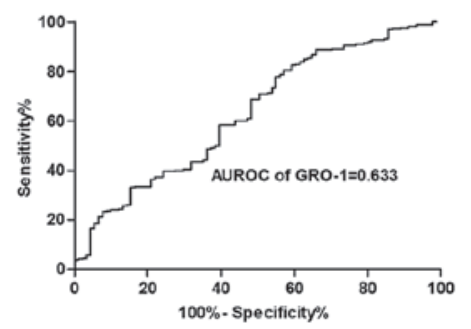

G

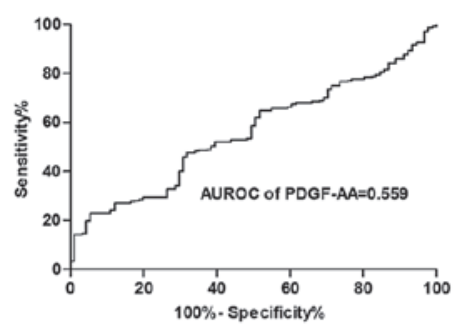

I

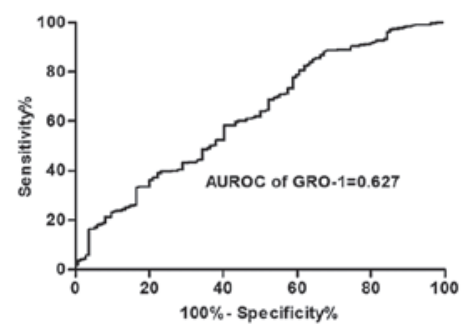

$\mathbf{K}$

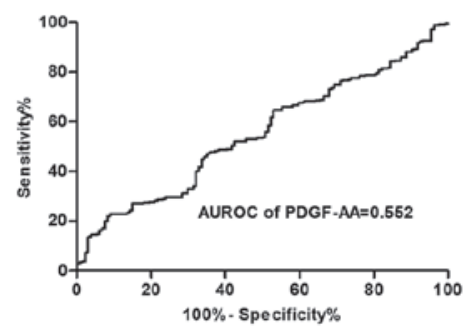

B

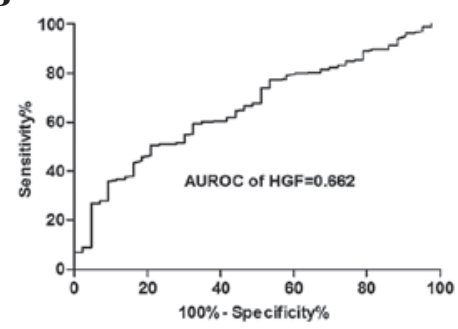

D

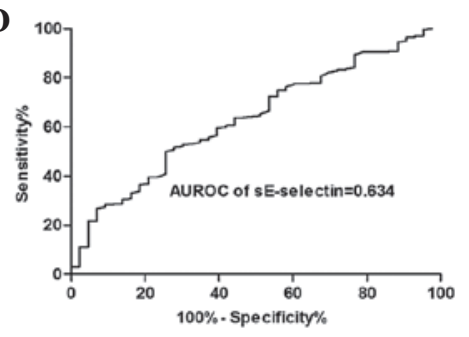

F

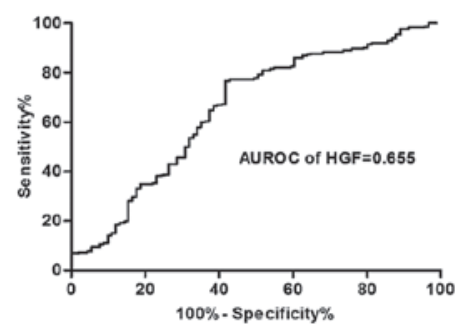

$\mathbf{H}$
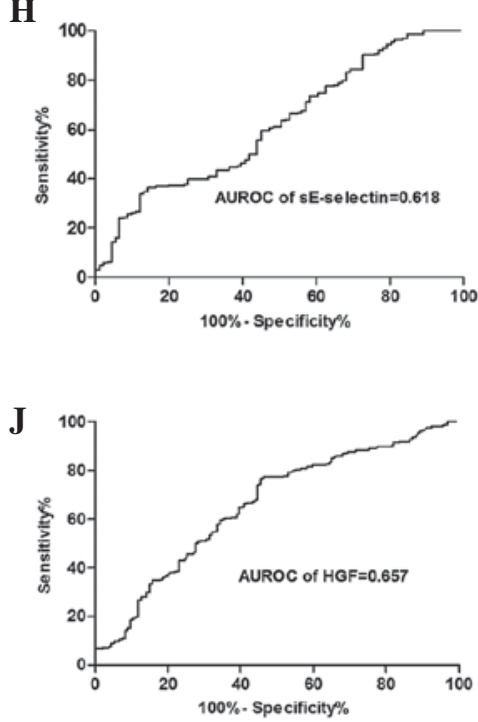

L

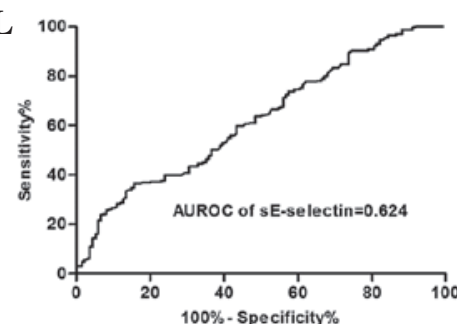

Figure 3. AUROC of GRO-1, HGF, PDGF-AA and sE-selectin for the diagnosis of CSCC patients. (A-D) CSCC patients were defined as the positive group and the CIN patients as the negative group. (E-H) CSCC patients were defined as the positive group and the healthy controls as the negative group. (I-L) The CSCC patients vs. the CIN patients and the healthy controls. CSCC, cervical squamous cell carcinoma; CIN, cervical intraepithelial neoplasia; GRO-1, growth-regulated oncogene-1; HGF, hepatocyte growth factor; PDGF-AA, platelet-derived growth factor-AA; sE-selectin, soluble E-selectin; AUROC, areas under the receiver operating characteristic curve. 
Table V. Sensitivity and specificity of the optimal cut-off values in different screening groups.

\begin{tabular}{|c|c|c|c|c|}
\hline Group & GRO-1 & HGF & PDGF-AA & sE-selectin \\
\hline \multicolumn{5}{|l|}{ CSCC vs CIN } \\
\hline Cut-off point (pg/ml) & 902.27 & 717.72 & 8287.73 & 22176.68 \\
\hline Sensitivity (\%) & 39.04 & 50.68 & 78.77 & 50.00 \\
\hline Specificity (\%) & 81.40 & 79.07 & 32.56 & 74.42 \\
\hline \multicolumn{5}{|c|}{ CSCC vs healthy controls } \\
\hline Cut-off point (pg/ml) & 466.89 & 494.00 & 19898.33 & 27399.83 \\
\hline Sensitivity (\%) & 80.48 & 76.71 & 22.95 & 36.64 \\
\hline Specificity $(\%)$ & 42.86 & 58.24 & 94.51 & 85.71 \\
\hline \multicolumn{5}{|c|}{ CSCC vs CIN and healthy controls } \\
\hline Cut-off point (pg/ml) & 407.58 & 494.00 & 19898.33 & 27399.83 \\
\hline Sensitivity $(\%)$ & 88.01 & 76.71 & 22.95 & 36.64 \\
\hline Specificity (\%) & 32.84 & 54.48 & 91.04 & 84.33 \\
\hline
\end{tabular}

GRO-1, growth-regulated oncogene-1; HGF, hepatocyte growth factor; PDGF-AA, platelet-derived growth factor-AA; sE-selectin, soluble E-selectin; CSCC, cervical squamous cell carcinoma; CIN, cervical intraepithelial neoplasia.

A

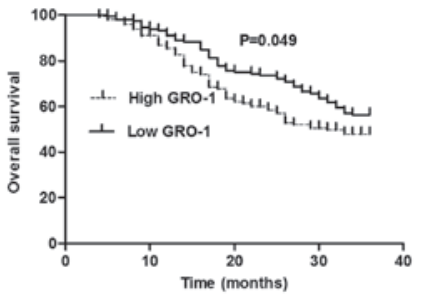

C

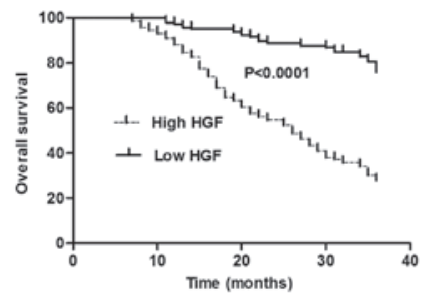

E

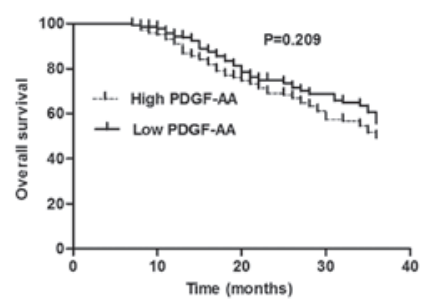

G

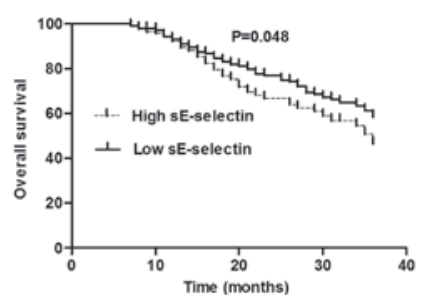

B

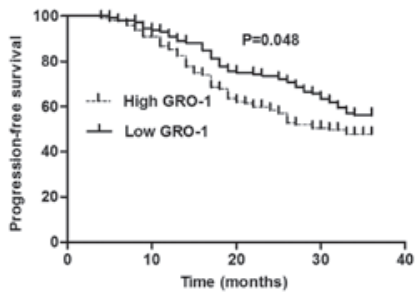

D

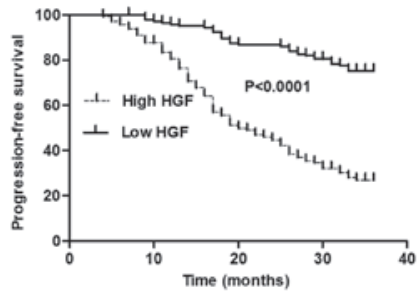

F

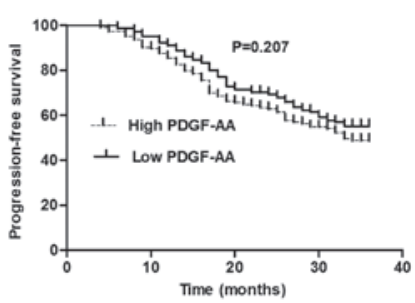

H

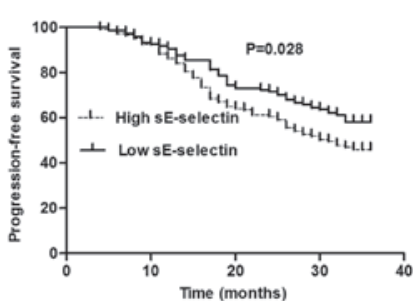

Figure 4. Kaplan-Meier plots of GRO-1, HGF, PDGF-AA and sE-selectin in CSCC patients. (A-B) Survival curves of GRO-1. (C-D) Survival curves of HGF. (E-F) Survival curves of PDGF-AA. (G-H) Survival curves of sE-selectin. CSCC, cervical squamous cell carcinoma; GRO-1, growth-regulated oncogene-1; HGF, hepatocyte growth factor; PDGF-AA, platelet-derived growth factor-AA; sE-selectin, soluble E-selectin. 
Table VI. Multivariate Cox regression in cervical squamous cell carcinoma patients.

\begin{tabular}{|c|c|c|c|c|c|c|}
\hline \multirow[b]{2}{*}{ Risk factor } & \multicolumn{3}{|c|}{ OS } & \multicolumn{3}{|c|}{ PFS } \\
\hline & P-value & HR & $95 \% \mathrm{CI}$ & P-value & HR & $95 \% \mathrm{CI}$ \\
\hline Age: $>45$ vs $\leq 45$ years & 0.277 & 1.235 & $0.844-1.808$ & 0.237 & 1.258 & $0.860-1.840$ \\
\hline FIGO stage: III+IV vs I+II & $<0.0001$ & 2.616 & $1.727-3.961$ & $<0.0001$ & 2.540 & $1.683-3.835$ \\
\hline Metastasis: Positive vs negative & 0.911 & 0.967 & $0.534-1.752$ & 0.985 & 0.994 & $0.548-1.804$ \\
\hline Grading: 3 vs $1+2$ & 0.316 & 0.816 & $0.549-1.214$ & 0.293 & 0.808 & $0.544-1.202$ \\
\hline Pelvic nodal status: Positive vs negative & 0.001 & 1.950 & $1.320-2.881$ & 0.001 & 1.982 & $1.342-2.927$ \\
\hline Tumor size: $>4 \mathrm{~cm}$ vs $\leq 4 \mathrm{~cm}$ & 0.676 & 0.923 & $0.633-1.345$ & 0.668 & 0.921 & $0.631-1.343$ \\
\hline GRO-1 (pg/ml): High vs low levels & 0.554 & 1.117 & $0.774-1.614$ & 0.627 & 1.095 & $0.759-1.579$ \\
\hline HGF (pg/ml): High vs low levels & $<0.0001$ & 5.640 & $3.596-8.846$ & $<0.0001$ & 5.279 & $3.368-8.276$ \\
\hline PDGF-AA (pg/ml): High vs low levels & 0.187 & 0.776 & $0.533-1.130$ & 0.206 & 0.785 & $0.540-1.142$ \\
\hline sE-selectin (pg/ml): High vs low levels & 0.695 & 0.929 & $0.643-1.342$ & 0.977 & 0.995 & $0.690-1.433$ \\
\hline HPV: Positive vs negative & 0.301 & 1.251 & $0.819-1.910$ & 0.260 & 1.276 & $0.835-1.948$ \\
\hline
\end{tabular}

PFS, progression-free survival; OS, overall survival time; HR, hazard ratio; CI, confidence interval; GRO-1, growth-regulated oncogene-1; HGF, hepatocyte growth factor; PDGF-AA, platelet-derived growth factor-AA; sE-selectin, soluble E-selectin.

A

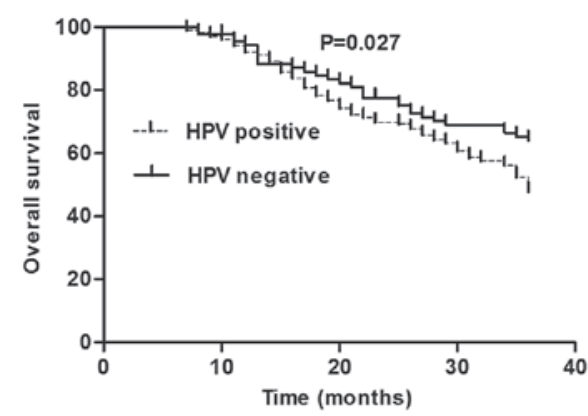

B

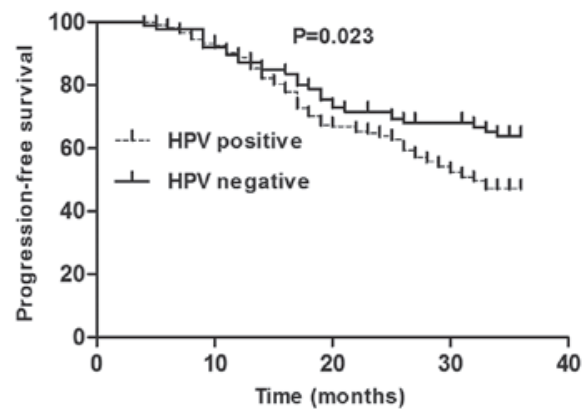

Figure 5. Kaplan-Meier plots of HPV in cervical squamous cell carcinoma patients. (A and B) Survival curves of HPV. HPV, human papillomavirus.

demonstrate an association between sE-selectin and lymph node metastasis (33). Other studies indicated that sE-selectin is not able to predict the metastasis of colorectal cancer (34). Therefore, this requires further investigation. It was also observed that the levels of sE-selectin were significantly associated with age, which is in accordance with a previous study (35). This may owe to a more active metabolic activity in young patients. No significant correlation was identified between sE-selectin and grading.

In the analysis of high-risk HPV infection in CSCC patients, a higher rate of infection was found in the CSCC patients compared with the CIN patients illustrating that HPV infection is closely associated with the occurrence of cervical cancer $(3,4)$. However, no significance was found between HPV infection and clinicopathological variables, which is consistent with the study by Bachtiary et al (36). The association between HPV and the four biomarkers was also analyzed and only the serum levels of HGF in HPV-positive patients were higher than HPV-negative patients. Walker et al (37) clarified that the overexpression of HGF is closely associated with cervical HPV infection.

The association between serum levels of GRO-1, HGF, PDGF-AA and sE-selectin in patients with CSCC was inves- tigated in the present study, and it was revealed that HGF was associated with the other three factors, and PDGF-AA was associated with GRO-1. Certain studies have demonstrated that HGF can enhance the generation of GRO-1 and VEGF in vitro (38) and another study indicated that serum levels of HGF in head and neck squamous cell carcinoma can regulate the expression of PDGF-A (39). There were correlations between two of either GRO-1, HGF and PDGF-AA in the present study, which suggests that they have a common role in angiogenesis $(11,17,40)$, while no association was found between sE-selectin and GRO-1 and PDGF-AA. Studies investigating this issue are rare and thus further investigation is required.

The diagnostic value of the four biomarkers in CSCC patients was also analyzed. HGF obtained the highest diagnostic value although the diagnostic value of all four factors was not significantly high. Hashem et al (41) reported the diagnostic value of HGF in prostate cancer, thus the detection of HGF in the serum of cervical cancer may provide aided diagnostic value.

Finally, the prognostic value of GRO-1, HGF, PDGF-AA, sE-selectin and HPV was examined in patients with CSCC. The results indicated that patients with a low expression of GRO-1, HGF and sE-selectin had significantly longer 
OS and PFS than those with high expression of the three factors. Furthermore, the HPV-positive patients had shorter OS and PFS compared with the HPV-negative patients, but only the levels of HGF, FIGO stage and pelvic lymph node metastasis were independent prognostic factors in the multivariate Cox analysis. Cheng et al (42) reported that GRO-1 may be a promising adverse prognostic molecular marker, and Miyake et al (43) confirmed the poor prognostic value of GRO-1 in bladder cancer. Numerous studies have demonstrated the prognostic value of sE-selectin in colorectal cancer $(31,34)$. Our findings suggested that GRO-1 and sE-selectin may have an impact on the prognosis of CSCC patients by affecting FIGO stage or lymph node metastasis, thus GRO-1 and sE-selectin were not independent prognostic risk factors. Madsen et al (44) failed to verify the prognostic value of PDGF-AA in ovarian cancer. However, another study indicated the adverse prognosis of PDGF-AA in pancreatic cancer (26), thus the prognostic value of PDGF-AA in CSCC requires further investigation. However, the prognostic value of HPV remains controversial (6-10). Studies have reported the prognostic value of HGF in patients with head and neck squamous cell carcinoma and verified that the high expression of HGF was closely associated with a poor prognosis (45). Aune et al (25) demonstrated its adverse prognostic significance in ovarian cancer. In cervical cancer, the prognostic value of the HGF receptor was clarified by Baykal et al (46), therefore, in addition to these traditional prognostic indicators, including FIGO stage and pelvic lymph node metastasis, the pre-treatment serum levels of HGF may be a predictor of tumor progression in certain early stage cervical carcinomas.

In conclusion, HGF may be a potential prognostic tumor marker rather than high-risk HPV types in patients with CSCC, therefore, detecting the serum levels of HGF may be useful for predicting the prognosis of CSCC patients.

\section{References}

1. Ferlay J, Shin HR, Bray F, et al: Estimates of worldwide burden of cancer in 2008: GLOBOCAN 2008. Int J Cancer 127: 2893-2917, 2010.

2. Wang SS, Sherman ME, Hildesheim A, Lacey JV Jr and Devesa S: Cervical adenocarcinoma and squamous cell carcinoma incidence trends among white women and black women in the United States for 1976-2000. Cancer 100: 1035-1044, 2004.

3. Walboomers JM, Jacobs MV, Manos MM, et al: Human papillomavirus is a necessary cause of invasive cervical cancer worldwide. J Pathol 189: 12-19, 1999.

4. Castellsagué X: Natural history and epidemiology of HPV infection and cervical cancer. Gynecol Oncol 110: S4-S7, 2008

5. Bao YP, Li N, Smith JS, Qiao YL and ACCPAB members: Human papillomavirus type distribution in women from Asia: a meta-analysis. Int J Gynecol Cancer 18: 71-79, 2008.

6. Schwartz SM, Daling JR, Shera KA, et al: Human papillomavirus and prognosis of invasive cervical cancer: a population-based study. J Clin Oncol 19: 1906-1915, 2001.

7. Kang WD, Kim CH, Cho MK, et al: HPV-18 is a poor prognostic factor, unlike the HPV viral load, in patients with stage IB-IIA cervical cancer undergoing radical hysterectomy. Gynecol Oncol 121: 546-550, 2011.

8. Riou G, Favre M, Jeannel D, Bourhis J, Le Doussal V and Orth G: Association between poor prognosis in early-stage invasive cervical carcinomas and non-detection of HPV DNA. Lancet 335: 1171-1174, 1990.

9. Higgins GD1, Davy M, Roder D, et al: Increased age and mortality associated with cervical carcinomas negative for human papillomavirus RNA. Lancet 338: 910-913, 1991.
10. Barreto CL, Martins DB, de Lima Filho JL and Magalhães V: Detection of human Papillomavirus in biopsies of patients with cervical cancer, and its association with prognosis. Arch Gynecol Obstet 288: 643-648, 2013.

11. Belperio JA, Keane MP, Arenberg DA, et al: CXC chemokines in angiogenesis. J Leukocyte Biol 68: 1-8, 2000.

12. Anisowicz A, Bardwell L and Sager R: Constitutive overexpression of a growth-regulated gene in transformed Chinese hamster and human cells. Proc Natl Acad Sci USA 84: 7188-7192, 1987.

13. Ogata H, Sekikawa A, Yamagishi H, et al: GRO $\alpha$ promotes invasion of colorectal cancer cells. Oncol Rep 24: 1479-1486, 2010.

14. Jung JJ, Noh S, Jeung HC, et al: Chemokine growth-regulated oncogene 1 as a putative biomarker for gastric cancer progression. Cancer Sci 101: 2200-2206, 2010.

15. Nakamura $T$, Nawa $K$ and Ichihara A: Partial purification and characterization of hepatocyte growth factor from serum of hepatectomized rats. Biochem Biophys Res Commun 122: 1450-1459, 1984

16. Cecchi F, Rabe DC and Bottaro DP: Targeting the HGF/Met signalling pathway in cancer. Eur J Cancer 46: 1260-1270, 2010.

17. Ferrara $\mathrm{N}$ and Kerbel RS: Angiogenesis as a therapeutic target. Nature 438: 967-974, 2005

18. Gogali A, Charalabopoulos K,Zampira I, et al: Soluble adhesion molecules E-cadherin, intercellular adhesion molecule-1, and E-selectin as lung cancer biomarkers. Chest 138: 1173-1179, 2010.

19. Allen C, Duffy S, Teknos T, et al: Nuclear factor- $\kappa$ B-related serum factors as longitudinal biomarkers of response and survival in advanced oropharyngeal carcinoma. Clin Cancer Res 13: 3182-3190, 2007.

20. Ogunwobi OO, Puszyk W, Dong HJ and Liu C: Epigenetic upregulation of HGF and c-Met drives metastasis in hepatocellular carcinoma. PloS One 8: e63765, 2013.

21. Tyan SW, Kuo WH, Huang CK, et al: Breast cancer cells induce cancer-associated fibroblasts to secrete hepatocyte growth factor to enhance breast tumorigenesis. PLoS One 6: e15313, 2011.

22. Horn LC, Hommel N, Roschlau U, Bilek K, Hentschel B and Einenkel J: Peritumoral stromal remodeling, pattern of invasion and expression of c-met/HGF in advanced squamous cell carcinoma of the cervix uteri, FIGO stages III and IV. Eur J Obstet Gynecol Reprod Biol 163: 76-80, 2012.

23. Grugan KD, Miller CG, Yao Y, et al: Fibroblast-secreted hepatocyte growth factor plays a functional role in esophageal squamous cell carcinoma invasion. Proc Natl Acad Sci USA 107: 11026-11031, 2010.

24. Nakamura T, Sakai K, Nakamura $T$ and Matsumoto $K$ : Anti-cancer approach with NK4: bivalent action and mechanisms. Anticancer Agents Med Chem 10: 36-46, 2010.

25. Aune G, Lian AM, Tingulstad S, et al: Increased circulating hepatocyte growth factor (HGF): a marker of epithelial ovarian cancer and an indicator of poor prognosis. Gynecol Oncol 121: 402-406, 2011

26. Rahbari NN, Schmidt T, Falk CS, et al: Expression and prognostic value of circulating angiogenic cytokines in pancreatic cancer. BMC cancer 11: 286, 2011.

27. Willenberg A, Saalbach A, Simon JC and Anderegg U: Melanoma cells control HA synthesis in peritumoral fibroblasts via PDGF-AA and PDGF-CC: impact on melanoma cell proliferation. J Invest Dermatol 132: 385-393, 2012.

28. Suzuki S, Dobashi Y, Hatakeyama Y, et al: Clinicopathological significance of platelet-derived growth factor (PDGF)-B and vascular endothelial growth factor-A expression, PDGF receptor- $\beta$ phosphorylation, and microvessel density in gastric cancer. BMC cancer 10: 659, 2010.

29. Donnem T, Al-Saad S, Al-Shibli K, Busund LT and Bremnes RM: Co-expression of PDGF-B and VEGFR-3 strongly correlates with lymph node metastasis and poor survival in non-small-cell lung cancer. Ann Oncol 21: 223-231, 2010.

30. Shikada Y, Yonemitsu Y, Koga T, et al: Platelet-derived growth factor-AA is an essential and autocrine regulator of vascular endothelial growth factor expression in non-small cell lung carcinomas. Cancer Res 65: 7241-7248, 2005.

31. Ferroni P, Roselli M, Spila A, et al: Serum sE-selectin levels and carcinoembryonic antigen mRNA-expressing cells in peripheral blood as prognostic factors in colorectal cancer patients. Cancer 116: 2913-2921, 2010. 
32. Dymicka-Piekarska V and Kemona H: Does colorectal cancer clinical advancement affect adhesion molecules (sP-selectin, sE-selectin and ICAM-1) concentration? Thromb Res 124 80-83, 2009.

33. Mantur M, Snarska J, Koper O, et al: Serum sICAM, sVCAM and $\mathrm{sE}$-selectin levels in colorectal cancer patients. Folia Histochem Cytobiol 47: 621-625, 2009.

34. Uner A, Akcali Z and Unsal D: Serum levels of soluble E-selectin in colorectal cancer. Neoplasma 51: 269-274, 2004.

35. Nash MC, Wade AM, Shah V and Dillon MJ: Normal levels of soluble E-selectin, soluble intercellular adhesion molecule-1 (sICAM-1), and soluble vascular cell adhesion molecule-1 (sVCAM-1) decrease with age. Clin Exp Immunol 103: 167-170, 1996.

36. Bachtiary B, Obermair A, Dreier B, et al: Impact of multiple HPV infection on response to treatment and survival in patients receiving radical radiotherapy for cervical cancer. Int J Cancer 102: 237-243, 2002

37. Walker F, Kermorgant S, Daraï E, et al: Hepatocyte growth factor and c-Met in cervical intraepithelial neoplasia overexpression of proteins associated with oncogenic human papillomavirus and human immunodeficiency virus. Clin Cancer Res 9: 273-284, 2003.

38. Dong G, Lee TL, Yeh NT, et al: Metastatic squamous cell carcinoma cells that overexpress c-Met exhibit enhanced angiogenesis factor expression, scattering and metastasis in response to hepatocyte growth factor. Oncogene 23: 6199-6208, 2004.
39. Worden B, Yang XP, Lee TL, et al: Hepatocyte growth factor/scatter factor differentially regulates expression of proangiogenic factors through Egr-1 in head and neck squamous cell carcinoma. Cancer Res 65: 7071-7080, 2005.

40. Carmeliet P and Jain RK: Angiogenesis in cancer and other diseases. Nature 407: 249-257, 2000.

41. Hashem M and Essam T: Hepatocyte growth factor as a tumor marker in the serum of patients with prostate cancer. J Egypt Natl Canc Inst 17: 114-120, 2005.

42. Cheng WL, Wang CS, Huang YH, et al: Overexpression of CXCL1 and its receptor CXCR2 promote tumor invasion in gastric cancer. Ann Oncol 22: 2267-2276, 2011.

43. Miyake M, Lawton A, Goodison S, et al: Chemokine (CXC) ligand 1 (CXCL1) protein expression is increased in aggressive bladder cancers. BMC cancer 13: 322, 2013.

44. Madsen CV, Steffensen KD, Olsen DA, et al: Serial measurement of serum PDGF-AA, PDGF-BB, FGF2, and VEGF in multiresistant ovarian cancer patients treated with bevacizumab. J Ovarian Res 5: 23, 2012.

45. Rischin D, Fisher R, Oliner K, et al: Prognostic significance of interleukin-8 (IL-8) and hepatocyte growth factor (HGF) in patients with head and neck squamous cell carcinoma (HNSCC) treated with chemoradiation on a phase III trial. J Clin Oncol (Meeting Abstracts) 28: 5509, 2010.

46. Baykal C, Ayhan A, Al A, Yüce K and Ayhan A: Overexpression of the c-Met/HGF receptor and its prognostic significance in uterine cervix carcinomas. Gynecol Oncol 88: 123-129, 2003. 